| Revista: Ciencia, Tecnología y Política | Año 1 Número 1 | 2018 | www.revistas.unlp.edu.ar/CTyP |

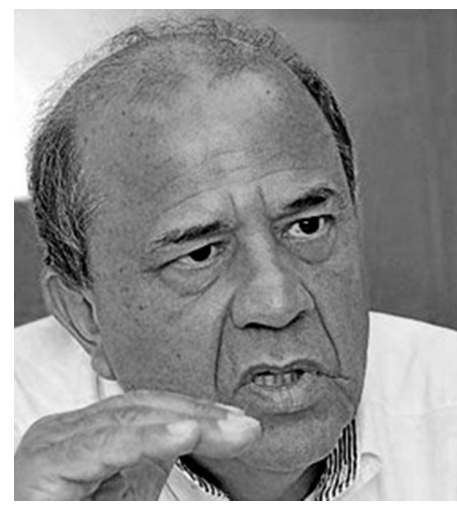

Renato Dagnino

Profesor Titular en el Departamento de Política Científica y Tecnológica de la Universidad de Campinas (UNICAMP). Se especializa en el estudio de las relaciones Ciencia -Tecnología-Sociedad en América Latina.

rdagnino@ige.unicamp.br

\title{
Elementos para una Política Cognitiva popular y soberana
}

Resumen: Las políticas de Educación y de Ciencia, Tecnología e Innovación, que llamaremos Política Cognitiva deberían ser pensadas en los países periféricos como un todo sistémico. Estas políticas que deberían impulsar y desarrollar los movimientos sociales, populares y de izquierda, requieren un significativo cambio del marco analíticoconceptual, que sea coherente con la magnitud de las transformaciones que se pretenden y que nuestras sociedades requieren. La dimensión de este desafío en los planos económico, social, político, ambiental y de recursos naturales, nos exige una conducta semejante a la que adoptan los países de capitalismo avanzado para establecer las metas de su llamada "sociedad del conocimiento". En este trabajo se describen algunos elementos de diagnóstico de situación a escala global, a tener en cuenta, para la elaboración de una nueva política cognitiva. Se explicitan los desafíos que un marco analíticoconceptual contra-hegemónico debería tener en cuenta y se discuten cursos de acción para alcanzar las metas estratégicas propuestas

Palabras clave: Política cognitiva, dinámica tecnocientífica global, economía solidaria, tecnología social, política científica, política educativa
Elements for a popular and sovereign Cognitive Policy

Abstract: The policies of Education, Science, Technology and Innovation, which we will call Cognitive Policy, should be thought of in the peripheral countries, as a systemic whole. These policies that should promote and develop social, left and popular movements, require a significant change in the analyticalconceptual framework, which is consistent with the magnitude of the transformations that are intended and that our societies require. The dimension of this challenge at the economic, social, political, environmental and natural resources levels, requires a behavior similar to that adopted by advanced capitalist countries to establish the goals of their so-called "knowledge society". This paper describes some elements of diagnosis on a global scale, to be taken into account, for the elaboration of a new cognitive policy. The challenges that an analytical-conceptual and counter-hegemonic framework should take into account and courses of action to reach the strategic goals proposed are explained.

Keywords: Cognitive policy, global technoscientific dynamics, solidarity economy, social technology, educational policy, scientific policy 


\section{Introducción}

Las políticas Educativas y de Ciencia, Tecnología e Innovación que deberían impulsar y desarrollar los movimientos sociales, populares y de izquierda requieren un significativo cambio del marco analítico-conceptual, que sea coherente con la magnitud de las transformaciones que se pretenden y que nuestras sociedades requieren. La dimensión de este desafío en los planos económico, social, político, ambiental y de recursos naturales, nos exige una conducta semejante a la que adoptan los países de capitalismo avanzado para establecer las metas de su llamada "sociedad del conocimiento".

El neoliberalismo, al establecer como verdad que la innovación se hace en las empresas y que ademas, vía derrame, son ellas las que posibilitan el desarrollo social, agregó oportunistamente -et pour cause- el término innovación a lo que se trataba como política de Ciencia y Tecnología, dando origen a la expresión política de Ciencia, Tecnología e Innovación (PCTI). A medida que esta expectativa de "derrame" se frustró, la PCTI pasó a ser tratada en conjunto con la política de educación, ya que debido a que funcionan como políticas-medio, son las responsables de viabilizar las políticas-fin que abarcan, en cascada, políticas sectoriales y sociales referentes a los planes, condiciones y oportunidades que permiten alcanzar las metas estratégicas globales.

En el caso de nuestros países, las políticas de Ciencia, Tecnología, Innovación y de Educación, en especial la de educación superior, a pesar de seguir formalmente separadas debido a nuestra arquitectura institucional, deberían ser pensadas como un todo sistémico. Por eso de ahora en más las Ilamaremos Política Cognitiva.

Otro elemento a tener en cuenta es que cada vez es más inadecuada la diferenciación entre investigación científica y desarrollo tecnológico. De hecho, los dos recortes -espacial y temporal- tradicionalmente empleados para distinguirlos y separar ciencia y tecnología, son obsoletos frente a la realidad observada. El 70\% de los recursos asignados a la investigación en el mundo se gastan en empresas (y el 70\% de estos en compañías multinacionales). El 30\% restante, que se gasta en instituciones públicas, está claramente sometido a los intereses empresariales. Mantener esa separación limita la acción de los gobiernos; sobre todo de aquellos que se orientan al cambio social y económico. Por esta razón se adopta aquí el concepto de Tecnociencia para dar cuenta y enfatizar esa convergencia. Además, es muy probable que aquella separación (ciencia, de un lado, y tecnología, del otro) haya sido una manipulación ideológica del capital para hacernos creer que hay algo intrínsecamente verdadero y bueno -la ciencia- que puede ser "usado" para el "bien" o para el "mal" -la tecnología-. Falacia que encubre que los valores e intereses del capital están impregnados en el conocimiento tecnocientífico.

En este contexto, desarrollar un nuevo marco analítico-conceptual para un proyecto popular y de izquierda involucra establecer diagnósticos y desafíos para el futuro y cursos de acción bien distintos de los formulados hasta ahora. 
| Revista: Ciencia, Tecnología y Política | Año 1 Número 1 | 2018 | www.revistas.unlp.edu.ar/CTyP |

\section{Elementos de diagnóstico para una nueva Política Cognitiva}

Se describen a continuación algunos elementos de diagnóstico de situación, a escala global, a tener en cuenta, privilegiando contenidos relacionados con la Política Cognitiva:

1. La Dinámica Tecnocientífica global está crecientemente motorizada por las empresas transnacionales, que cada vez más controlan lo que aún se denomina investigación científica y que monopolizan lo que aún se designa por desarrollo tecnológico. Esta dinámica por lo tanto, lejos de ser universal o neutra como insiste -miope o corporativamente- la élite científica, está crecientemente contaminada por los intereses y valores del capital.

2. Esa dinámica, en la que la "ciencia" y la "tecnología" se interpenetran sistemáticamente, es responsable del deterioro programado, la obsolescencia planificada, el consumismo exacerbado, la militarización imperialista, la degradación ambiental y por el consiguiente agravamiento de la desigualdad económica y de la injusticia social.

3. A diferencia de la visión ideológica hegemónica difundida por la élite (o corporación) científica, hay argumentos lógicos e históricos y evidencias empíricas suficientes para demostrar que no existe una "ciencia" buena, verdadera y éticamente neutra pasible de ser usada, mediante el desarrollo de tecnología, para el "bien" o para el "mal". Esta visión ha sido cuestionada en los países de capitalismo avanzado y también en América Latina desde 1970, originando el campo de los estudios sociales de CyT o estudios sobre Ciencia, Tecnología y Sociedad (CTS). Su vertiente radical, al dedicarse a la deconstrucción de los mitos de la neutralidad y del determinismo, presentes incluso en la concepción marxista ortodoxa, viene encontrando creciente aceptación en la comunidad latinoamericana de investigación en este campo.

4. La evidencia empírica global muestra que, contrariamente a lo que difunde la élite científica, la conveniencia de colocar la investigación realizada en la universidad al servicio del lucro (o "competitividad") de la empresa, no beneficia -ni cognitiva ni económicamente- a ninguno de estos dos actores. Por un lado, en todo el mundo la importancia del resultado de la investigación universitaria para las empresas es muy pequeña. En los EE.UU., por ejemplo, sólo el 1\% del gasto en investigación de las empresas se destina a proyectos que involucran universidades o institutos de investigación. Esto no significa que los resultados de la investigación en la universidad no sean esenciales para la innovación y la competitividad de las empresas estadounidenses. La importancia está en el personal entrenado en investigación en la universidad que participa en la I+D empresarial. De hecho, más de la mitad de los magísteres y doctores formados en "ciencias duras" en las universidades son contratados por las empresas para realizar I+D. Sin embargo, esto no ocurre así en los países periféricos donde las empresas no aprovechan a los posgraduados para innovar. En el caso de Brasil, esta situación nada tiene que ver con el supuesto retraso de los empresarios "brasileños", ya que la mayoría son de firmas multinacionales que operan en ramas de alta intensidad tecnológica. Estas empresas en sus países 
de origen tienen que hacer I+D para innovar, pero en Brasil, al igual que las empresas de capital nacional, "innovan" comprando máquinas y equipos. La ancestral dependencia cultural y la adopción de un modelo eurocéntrico de organización social -característica de nuestra condición periférica- hace que prácticamente todo lo que se fabrica aquí en el "Sur", en la periferia del capitalismo, ya fue producido en el "Norte". Las empresas locales prefieren innovar a través de la adquisición de tecnología ya desarrollada; en especial la incorporada en máquinas y equipamientos (como afirman el $80 \%$ de las empresas llamadas innovadoras).

Por otro lado, la inversión del Estado en CTI en relación al PBI en nuestros países, ha sido históricamente mucho más importante (en comparación con los países de capitalismo avanzado) que el gasto privado. En el caso de Brasil, a pesar de los recursos que desde hace más de seis décadas se asigna al fomento de la relación universidad-empresa, su importancia para la estrategia innovadora de la empresa es muy pequeña. De las "empresas innovadoras brasileñas", sólo el $7 \%$ se involucra con universidades e institutos en busca de resultados de investigación; de ellas, el 70\% considera de baja importancia esa relación. Es posible, además, evidenciar para el caso brasileño, una disfuncionalidad de la PCTI que muy probablemente debe ocurrir en otros países periféricos. Entre 2006 y 2008 las empresas brasileñas aumentaron producción, ganancia y salarios, lo cual -en la lógica del capitalismo central globalizado- hubiera debido inducirlas a la innovación; a la vez, se formaron 90 mil magisteres en "ciencias duras" en todo el país, sin embargo muy pocos de ellos fueron contratados para hacer I+D en las empresas. Por el contrario, en los países centrales más de la mitad de los posgraduados (que son formados para realizar investigación en empresas) son contratados por ellas.

5. Los resultados sociotécnicos adscritos a la dinámica tecnocientífica global, aunque pudieran ser apropiados por los sectores populares y de izquierda, no serían capaces de materializar su proyecto político. Tampoco serían compatibles con el estilo de desarrollo que buscan. Más aún, ni siquiera posibilitarían sostener un desarrollo económico-social que evite, en los países periféricos, el salvajismo del capitalismo actual.

En el ámbito de la mayoría de las organizaciones populares y de izquierda, existe la idea de que la simple combinación o aggiornamiento del saber tradicional, ancestral, empírico o popular con el conocimiento tecnocientífico (entendido como científicamente verdadero y tecnológicamente eficiente, que debe ser "divulgado" y "extendido" al "hombre común"), es suficiente para generar formas de producción de bienes y servicios adecuados a su proyecto político. La noción de Adecuación Sociotécnica desarrollada por los seguidores del Pensamiento Latinoamericano de Ciencia, Tecnología y Sociedad, fue concebida buscando deshacer esa idea que, aunque atractiva y aparentemente justa y democrática, es simplista y tiende a ser perjudicial.

6. Los casos exitosos de desarrollo tecnocientífico en varios países periféricos ocurrieron en áreas donde no es posible (como decía Jorge Sabato) robar, copiar o comprar tecnología; y fueron financiados o fueron iniciativa del Estado. En el caso de Brasil algunos ejemplos son: la creación del Instituto 
Agronómico de Campinas y el Instituto Oswaldo Cruz a finales del siglo XIX, para combatir la plaga del café y la fiebre amarilla; la creación de la empresa estatal Embrapa para desarrollar tecnología inexistente que permitiera viabilizar las exportaciones del agronegocio; los programas de investigación de Petrobras para la extracción de petróleo en aguas profundas. Por otro lado, el esfuerzo desplegado para la formación de personal y los proyectos de investigación en las empresas Embraer, Telebras, Eletrobras y otras compañías estatales muestran que cuando una élite de poder económico o político presiona a través del Estado para que se desarrolle la tecnociencia demandada por sus intereses, ésta es generada. Lo mismo se verifica en el caso de Argentina donde ha sido el Estado y no el sector privado el responsable por las innovaciones y desarrollos autónomos realizados en las áreas nuclear, espacial, etc.

7. En las experiencias progresistas de la pasada década y media que tuvieron lugar en nuestro continente al contrario de lo que ocurrió con otras políticas sociales (mayor inclusión y distribución de la riqueza, derechos democráticos), que beneficiaron a los sectores populares y a las clases medias, la Política Cognitiva no logró una ruptura cualitativa con políticas previas.

En el caso de Brasil, al contrario de lo que ocurrió con otras políticas, las actividades de enseñanza e investigación financiadas con fondos públicos se mantuvieron distantes de las necesidades, intereses y demandas cognitivas de las mayorías. La comunidad de investigación, que ya había adoptado la meta suplementaria de la competitividad, derivada del neoliberalismo globalizante de finales de los años 1990, la siguió impulsando de forma hegemónica en el discurso de la Política Cognitiva. Paradójicamente, esta meta fue mantenida y ampliada por la coalición encabezada por el Partido de los Trabajadores. Con el propósito pragmático declarado de hacer de la competitividad una meta más útil, la élite científica la orientó a atender el interés de las empresas que, para llegar a ser competitivas, iban a innovar mediante la realización de I+D y, finalmente, vía desbordamiento o "derrame", promover el desarrollo. En ese contexto, aumentaron drásticamente los recursos asignados a la I+D y a las demás actividades innovadoras en las empresas. Se reforzó la idea, invalidada por la evidencia mundial, de que el aumento de la relación universidad-empresa, a través de la investigación universitaria orientada a las necesidades empresariales y la creación de incubadoras de empresas de base tecnológica creadas en las universidades, podrían contribuir a la competitividad de las empresas. Esa política fue un fracaso. Como dijimos anteriormente, las empresas localizadas en países periféricos innovan (y alcanzan altas ganancias) mediante la adquisición de nuevos equipamientos e insumos, desarrollados en las empresas de los países centrales.

\section{Desafíos}

En base a estos elementos de diagnóstico, mencionaremos los desafíos para el futuro que un marco analítico-conceptual contra-hegemónico debería identificar como foco de una nueva Política Cognitiva. Estos se destacan por su magnitud económica, relevancia social, importancia para la soberanía y la autonomía del país. Deberían tenerse en cuenta además para garantizar el acceso al gobierno -y la 
gobernabilidad posterior- de un proyecto popular y de izquierda.

Del lado de la "oferta cognitiva", es necesario cambiar la orientación de la investigación y de la formación de recursos humanos en las instituciones públicas. Hoy es fijada por el corporativismo de la élite científica y por el (des)interés de las empresas locales cuando se trata de absorber al personal formado.

Por otro lado, se debe privilegiar la adecuación sociotécnica de la tecnociencia convencional (concebida en función de la ganancia capitalista en el marco de la dinámica tecnocientífica global) con una nueva Política Cognitiva, económicamente sustentable, que amplíe las iniciativas de generación de trabajo y renta (y no de empleo y salario en empresas, como todavía se intenta sin éxito) en estructuras y organizaciones basadas en la propiedad colectiva de los medios de producción, en la autogestión y la solidaridad, particularmente en los emprendimientos solidarios.

Es evidente que la generación de un conocimiento tecnocientífico específico cuyo desarrollo necesita ser especialmente orientado, requerirá un cambio en el componente de políticas-medio de la PCTI. Esto no implica que sea sensato prescindir del potencial de investigación y del personal instalado en nuestras instituciones públicas de enseñanza e investigación. Con ese potencial deberá realizarse un monitoreo consciente y criterioso de las llamadas tecnologías emergentes (que emanan de la dinámica tecnocientífica global controlada por las compañías multinacionales y por los objetivos estratégicos de las grandes potencias) pero que pueden, si estuviesen enmarcadas en procesos de adecuación sociotécnica que involucren a los/las trabajadores/as, generar soluciones (que serán necesariamente originales e intensivas en conocimiento) para los grandes problemas nacionales.

Del lado de la "demanda cognitiva", se debería aprovechar esas iniciativas, mencionadas en la oferta cognitiva, mediante la concesión de recursos a las respectivas organizaciones y estructuras que las lleven adelante. De este modo se estará capacitando para desarrollar, en conjunto con instituciones públicas de enseñanza e investigación, procesos de adecuación sociotécnica que atiendan a sus especificidades e intereses.

Podría parecer contradictorio que en medio del descalabro económico y la crisis que han desatado los gobiernos de derecha de la región, en particular en Brasil y Argentina, y de la necesidad de derrotarlos retomando de inmediato el desarrollo con equidad, proponer una perspectiva tan radical para la elaboración de una Política Cognitiva soberana y transformadora como la que aquí se presenta. Sin embargo, dado que uno de sus resultados sería una tecnociencia capaz de viabilizar la Economía Solidaria mediante el desarrollo de la Tecnología Social, se puede comprender por qué esta perspectiva es coherente con un proyecto político popular y de izquierda.

Mirando hacia el futuro, es evidente que la dupla Economía Solidaria - Tecnología Social no se restringe a algo que sólo mejora la calidad de vida de los sectores populares. Se propone un socialismo 
fundamentado en la autogestión, en la propiedad colectiva de los medios de producción, en una concepción para ir "más allá del capital" atendiendo las cuestiones ambientales, de género, etc., y que por ello demanda un radical giro analítico-conceptual en la práctica de los científicos y tecnólogos politizados, comprometidos con los sectores populares.

\section{Cursos de acción}

Para alcanzar las metas estratégicas globales se proponen cuatro cursos de acción articulados:

1. Identificar y detallar las demandas cognitivas (económicas, sociales, políticas, ambientales) hasta el punto de hacer que sean transformadas en objeto de políticas y acciones de gobierno y en agendas de investigación y formación de personal de las instituciones públicas.

2. Es imprescindible en el transcurso de ese proceso hacer esfuerzos para estrechar el diálogo, involucrar y reforzar el poder de los integrantes de la comunidad de investigación que trabajan en las universidades e institutos de investigación públicos y que comparten un Proyecto Nacional popular y soberano. Con estos sectores, es necesario concebir agendas de investigación y enseñanza coherentes con las demandas cognitivas de ese proyecto, que además incorporen el conocimiento ancestral y popular que poseen los/las trabajadores/as. Estas agendas deben implementarse mediante las condiciones humanas, materiales y financieras que se requieran.

3. Identificar, entre las más importantes acciones de gobierno, aquellas que pueden llevarse a cabo a través de la movilización de las 3/4 partes de la población en edad de trabajar que no tiene (y probablemente nunca tendrá) un empleo formal. Este sector, que las empresas no tienen condiciones o voluntad de emplear, puede encontrar oportunidades de trabajo y renta en la Economía Solidaria, protegidos en parte de la explotación capitalista. Para ello, se deben fomentar los emprendimientos solidarios y los procesos de adecuación sociotécnica de los conocimientos proveniente de la dinámica tecnocientífica global y de desarrollo de Tecnología Social. Estos deberán contar con la participación de las instituciones públicas y recibir financiamiento en calidad y cantidad compatibles con la importancia de este curso de acción. La PCTI deberá estar articulada con la sostenibilidad económica de estos emprendimientos, con apoyo mediante subsidio directo a la organización y consolidación de sus cadenas de producción y consumo de bienes y servicios, y con la asignación del poder adquisitivo del Estado para que la población tenga acceso a ellos.

4. Se deberá apoyar en las instituciones públicas el monitoreo y la realización de actividades de investigación y formación de personal específicamente orientado a la aplicación de esta nueva Política Cognitiva popular, transformadora y soberana. 